\title{
PHOTOPOLARIMETRY OF PLANETS
}

\section{T. GEHRELS}

The University of Arizona, Tucson, Ariz., U.S.A.

\begin{abstract}
Polarimetry is reviewed in a new book of which I am the editor (Gehrels 1974) while detailed papers of our group in Arizona continue to appear in a series
\end{abstract} 'The Wavelength Dependence of Polarization' in the Astronomical Journal.

The PIONEER missions provide the first encounter of Jupiter on 4 December 1973. Our 2.5-cm Maksutov $F / 3.4$ telescope has three square focal-plane diaphragms: $2: 3$ for zodiacal light, 0.5 for photopolarimetry and 0.03 for imaging of Jupiter and satellites. There are two spectral bands, namely $390-500 \mathrm{~nm}$ and $595-720 \mathrm{~nm}$. Weinberg and Hanner (1973) are the investigators for the zodiacal light. The instrument was built by Santa Barbara Research Center (Pellicori et al., 1973); specifications have also been published by KenKnight (1971). Maps of brightness and linear polarization of Jupiter are to be made during encounter at about $40^{\circ}, 100^{\circ}$, and $140^{\circ}$ phase angle. We expect to determine aerosol characteristics and the amount of gas above the clouds. We also plan to observe the Galilean satellites at about $20^{\circ}, 60^{\circ}$ and $120^{\circ}$ phase. The Pioneer spacecraft spins at $5 \mathrm{rpm}$ and the imaging is done by compositing consecutive scans on the planetary disk (Gehrels et al., 1972). The images will be rectified for changing geometry, during the hour needed for a picture, and other image enhancement techniques will be applied. The images will have resolution somewhat higher than those obtained from Earth, but the precise increase depends on uncertain flight hazards, e.g. the radiation belts. Groundbased observational programs of Jupiter during 1973 and 1974 are needed for tie-in with the Pioneer measurements. The Pioneer program is conducted by the Ames Research Center (see NASA SP-268). My coinvestigators at the University of Arizona are D. L. Coffeen, C. E. KenKnight, W.Swindell, and M. G. Tomasko.

\section{References}

Gehrels, T. (ed.): 1974, Planets, Stars and Nebulae Studied with Photopolarimetry, The University of Arizona Press, Tucson, Ariz.

Gehrels, T., Suomi, V. E., and Krauss, R. J.: 1972, 'The Capabilities of the Spin-Scan Imaging Techniques', in Space Research XII, Akademie-Verlag, Berlin, p. 1765.

Hanner, M. S. and Weinberg, J. L.: 1973, Sky Telesc. 45, 217.

KenKnight, C. E.: 1971, 'Physical Studies of Minor Planets', in NASA SP-267, p. 633.

NASA SP-268, 'The Pioneer Mission to Jupiter', National Aeronautics and Space Administration, Washington.

Pellicori, S. F., Russell, E. E., and Watts, L. A.: 1973, Appl. Opt. 12, 1246.

\section{DISCUSSION}

Irvine: Are there new data on the center-to-limb variation of polarization for Jupiter? This is important for comparison with the center-to-limb variation of equivalent widths of absorption lines, such as those of methane and ammonia.

Gehrels: Yes, J. S. Hall and L. Riley have recently published, in the Lowell Obs. Bull., detailed polarization measurements made with an area scanner. 